\title{
THE RELATIONSHIP BETWEEN PULSE PRESSURE AND INFLAMMATION WITH LEFT \\ VENTRICULAR DIASTOLIC DYSFUNCTION IN CHRONIC KIDNEY DISEASE PATIENTS
}

Kenneth Yong FRACP ${ }^{1,2}$, Trevor Mori B Sc PhD ${ }^{1}$, Gerard Chew FRACP ${ }^{1}$, Lawrence J Beilin PhD ${ }^{1}$, Ian

Puddey $\mathrm{PhD}^{1}$, Gerald Watts $\mathrm{PhD}^{1}$, Gursharan Dogra PhD ${ }^{3}$, Neil Boudville FRACP ${ }^{1,3}$, Wai Lim PhD ${ }^{1,3}$

${ }^{1}$ School Of Medicine And Pharmacology, University Of Western Australia, Perth Australia

${ }^{2}$ Department Of Nephrology, Prince Of Wales Hospital, Sydney Australia

${ }^{3}$ Department Of Nephrology, Sir Charles Gairdner Hospital, Perth Australia

Short Title: $\quad$ Inflammation And Diastolic Dysfunction In Chronic Kidney Disease

Financial Support: Jacquot Research Establishment Award

Jacquot Research Entry Scholarship

National Health \& Medical Research Council Post-Graduate Scholarship

National Health \& Medical Research Council Project Grant (APP303151)

Pfizer CVL Grant

Corresponding Author

Dr. Kenneth Yong (MBBS, FRACP)

Department Of Nephrology

Level 3 High Street, Prince Of Wales Hospital

Randwick NSW 2031

Australia

kyonga08@yahoo.com.au

C

Disclosures: No financial or conflict of interests to declare

Abstract

Introduction

This article has been accepted for publication and undergone full peer review but has not been through the copyediting, typesetting, pagination and proofreading process, which may lead to differences between this version and the Version of Record. Please cite this article as doi: 10.1111/imj.14037 
Diastolic dysfunction (DD) is an important cause of cardiovascular disease (CVD) mortality in chronic kidney disease (CKD) patients. Non-traditional risk factors such as arterial stiffness and inflammation are implicated in the pathogenesis of DD in CKD patients.

Aim

To determine the association between inflammatory markers [interleukin(IL)-12, IL18, highly sensitive C-reactive protein (hsCRP)] and non-invasive markers of arterial stiffness [24-hour pulse pressure (PP)] with DD in stage 3-4 CKD patients.

\section{Methods}

We performed a sub-analysis of 78 non-diabetic stage 3-4 CKD subjects to determine the relationship between 24-hour PP, IL-12, IL-18 and hsCRP with DD.

Results

DD was present in 38 subjects (49\%). Subjects with DD were significantly older $(61.0 \pm 1.9$ vs $50.2 \pm 2.0$ years; $p<0.001)$ and had higher 24 -hour PP $[48(95 \% \mathrm{Cl} 45,52)$ vs $43(95 \% \mathrm{Cl} 41,45) \mathrm{mmHg} ; \mathrm{p}<0.005]$. 24-hour PP was associated with DD ( $p=0.02)$ but this was no longer significant after adjustment for age $(p=0.31)$. Serum IL-12, IL-18 and hsCRP levels were not significantly different between subjects with or without DD.

\section{Conclusion}

Asymptomatic subclinical DD was present in $50 \%$ of a cohort of stage $3-4$ CKD patients but was not associated with IL-12, IL-18 or hsCRP. The association between 24-hour PP and DD was no longer apparent following adjustment for age but given the small sample size our findings will need to be explored in larger sized cohorts of individuals with moderate stage CKD. 


\section{Background}

Patients with chronic kidney disease (CKD) have a 30-fold increased risk of ageadjusted cardiovascular disease (CVD) mortality in comparison to the general population ${ }^{1,2}$. Although atherosclerosis is the predominant cause of CVD-related mortality in the general population, non-atherosclerotic CVD such as cardiac failure and sudden cardiac death (SCD) are responsible for approximately $30 \%$ of CVD-related mortality in CKD patients ${ }^{3}$.

Left ventricular diastolic dysfunction (DD) is an early manifestation of nonatherosclerotic CVD which precedes clinically overt cardiac failure in CKD patients ${ }^{4,5}$. DD is more common than abnormal systolic function in CKD patients as demonstrated by the Chronic Renal Insufficiency Cohort (CRIC) study of 3939 stage 3-4 CKD patients in which 75\% and $12 \%$ displayed echocardiographic evidence of DD and systolic dysfunction respectively ${ }^{6}$. CKD patients are also at increased risk for DD with an approximately 3-fold increased prevalence of severe DD (pseudonormalisation and restrictive filling patterns) in comparison to the general population ${ }^{7,8}$. Severe DD is an independent poor prognostic marker and is associated with a 10- and 2.9-fold increased risk of all-cause mortality in the general population and CKD patients respectively ${ }^{7,8}$.

The underlying pathology of DD involves myocardial fibrosis which results in reduced cardiac compliance and manifests clinically as intolerance to even small changes in blood volume or other haemodynamic stressors ${ }^{5}$. The pathogenesis of myocardial fibrosis is not completely understood particularly in CKD patients in whom cardiac mass and fibrosis is significantly increased in comparison to age-matched healthy subjects or those with diabetes or hypertension ${ }^{9}$. Both traditional risk factors such as diabetes or hypertension and non-traditional risk factors such as arterial stiffness and chronic inflammation are likely to be involved in the pathogenesis of DD but the relative magnitude of their respective contributions to DD remains unclear in CKD patients ${ }^{5,10-12}$.

Arterial pulse pressure (PP) and highly sensitive C-reactive protein (hsCRP) are surrogate markers of arterial stiffness and inflammation respectively which are associated with increased CVD mortality in CKD patients ${ }^{13,14}$. Recent studies have suggested that serum hsCRP levels and PP index may be associated with increased risk of DD in CKD patients ${ }^{6,15}$. 
Interleukin(IL)-12 and IL-18 are pro-inflammatory cytokines implicated in the development of atherosclerosis and are predictive of coronary events and CVD mortality in the general population $^{16-18}$. CKD patients have 2-3 fold elevations in serum IL-12 and IL-18 levels compared to the general population ${ }^{19}$. Some but not all studies have suggested an association between IL-12 and IL-18 in CKD patients with CVD surrogate markers and allcause mortality but their relationship with DD has not previously been explored ${ }^{19-21}$.

We hypothesised that increased serum concentrations of IL-12, IL-18, hsCRP and 24hour PP are associated with DD in CKD patients. The primary aim of this study was to evaluate the association between IL-12, IL-18, hsCRP and 24-hour PP with DD in stage 3-4 CKD patients. 


\section{Methods}

This was a sub-analysis of prospectively obtained samples from 78 participants in the Fish-CoQ Study (ACTRN12605000088640), a double-blinded randomised placebo-controlled trial examining the effects of fish oil and coenzyme Q10 on blood pressure and lipid profile in stage 3-4 CKD patients (Figure 1). The study design, methods, inclusion and exclusion criteria have previously been published ${ }^{22}$. Participants were recruited from nephrology units of 3 major tertiary hospitals in Perth, Western Australia. The main inclusion criteria were age between 25 and 75 years, serum creatinine $<350 \mu \mathrm{mol} / \mathrm{L}$ and estimated glomerular filtration rate (eGFR) $15-60 \mathrm{~mL} / \mathrm{min} / 1.73 \mathrm{~m}^{2}$ by the Modification of Diet in Renal Disease (MDRD) equation ${ }^{23}$. Exclusion criteria were the presence of diabetes, current smokers, major surgery or any CVD event within 3 months prior to randomisation, blood pressure exceeding $170 / 100 \mathrm{mmHg}$, liver disease, nephrotic syndrome (proteinuria quantified as over $3 \mathrm{~g} /$ day or spot urine protein:creatinine ratio above $300 \mathrm{mg} / \mathrm{mmol}$ ) and use of non-steroidal inflammatory or immunosuppressive drugs. Patients with previous CVD or those receiving anti-hypertensives or lipid lowering agents including angiotensin converting enzyme (ACE) inhibitors, angiotensin receptor blockers (ARB) or statins were not excluded. Previous CVD was defined as previous angina, myocardial infarction, cerebrovascular accident, peripheral vascular disease or coronary artery disease demonstrated on coronary angiogram. The institutional ethics committees of Royal Perth Hospital, Sir Charles Gairdner Hospital and Fremantle Hospital in Perth, Western Australia approved the study and informed written consents were obtained from all participants (Ethics Approval Number: 2004/045).

\section{Exposure Factors}

The study exposure factors were pre-intervention mean 24-hour PP [difference between the mean 24-hour systolic blood pressure (SBP) and diastolic blood pressure (DBP)] and serum levels of IL-12, IL-18 and highly sensitive CRP (hsCRP). 


\section{Clinical Outcomes}

The primary outcome was the presence of pre-intervention DD. Secondary outcomes included the presence of pre-intervention severe DD (defined as grade II or III DD) and prespecified diastolic parameters of left atrial volume index (LAVI) and the ratio of early mitral inflow to mitral annular velocity (E/e' ratio).

\section{Measurement Of Pro-Inflammatory Cytokines}

Serum IL-12 and IL-18 levels were measured using enzyme-linked immunosorbent (ELISA) kits (BD Biosciences, California, USA) (MBL Co. Ltd, Nagoya, Japan) according to manufacturer's instructions with detection thresholds of $12.5 \mathrm{pg} / \mathrm{mL}$ and $7.8 \mathrm{pg} / \mathrm{mL}$, respectively. Intra- and inter-assay coefficient of variation for IL-12 and IL-18 were 7\% and $8 \%$, respectively. Serum hsCRP was measured using BNII Systems (Siemens Healthcare Diagnostics Inc. Network, DE 19714 USA) with detection threshold of $0.175 \mathrm{mg} / \mathrm{L}$.

Measurement Of 24-Hour Blood Pressure (BP) \& Pulse Pressure (PP)

Twenty-four hour ambulatory $\mathrm{BP}$ and heart rate $(\mathrm{HR})$ were obtained using the Spacelabs Monitor (Model 90217, SpaceLabs Medical Inc, Issaquah, Washington, USA) with measurements at 20- and 30-minute intervals during waking hours and sleep respectively. Mean 24-hour PP was determined as the difference between mean 24-hour SBP and DBP.

\section{Measurement Of Cardiac Parameters Using Echocardiography}

Baseline transthoracic echocardiograms were performed by two dedicated cardiac technicians at a single site (Hollywood Private Hospital, Perth, Australia) using the Acuson Sequioa Ultrasound System (Siemens, AG, Munich, Germany) with digital storage (KinetDx; Siemens AG). Measurements of cardiac volume, dimensions, systolic and diastolic function 
were performed according to standard guidelines from the American Society of Echocardiography ${ }^{24}$. Simpson's biplane method was used to calculate LV ejection fraction (LVEF). Left atrial volume was quantified by the Simpson's 2D biplane method and indexed for body surface area ${ }^{25}$. Pulsed wave Doppler techniques were used to measure pulmonary venous, transmitral inflow and mitral annular velocities in the apical four-chamber view. Data were collected as the mean of three measurements on separate cardiac cycles.

\section{Grading Of Diastolic Dysfunction}

DD was categorised as normal, grade I (impaired relaxation), grade II (pseudonormalisation) or grade III (restrictive) according to established criteria from the American Society of Echocardiography. Diagnosis of DD required the fulfilment of two or more following echocardiographic criteria based on the ratio of early and late mitral inflow velocity $(E / A)$, mitral annular velocity $\left(e^{\prime}\right)$, ratio of early mitral inflow to mitral annular velocity $\left(E / e^{\prime}\right)$, left atrial volume index (LAVI) and the difference between pulmonary venous atrial wave reversal duration and mitral $A$ wave duration $(\mathrm{Ar}-\mathrm{A})^{5,24}$.

\section{Biochemical Parameters}

Fasting serum creatinine $(\mu \mathrm{mol} / \mathrm{L})$, total cholesterol $(\mathrm{mmol} / \mathrm{L})$, low-density lipoprotein cholesterol (LDL-C; mmol/L), triglycerides (TG; $\mathrm{mmol} / \mathrm{L}$ ) and high-density lipoprotein cholesterol (HDL-C; mmol/L) levels were obtained from all participants ${ }^{22}$. Serum creatinine was measured by the Jaffe method ${ }^{26}$.

\section{Statistical Analysis}

Baseline characteristics are presented as mean \pm standard deviation (SD) for normally distributed data, geometric mean with $95 \%$ confidence interval $(95 \% \mathrm{Cl})$ for log transformed (base $e$ ) data and median (interquartile range; IQR) for skewed variables. Comparisons of continuous variables were examined using student's $t$-test or MannWhitney $U$ test where appropriate. Comparisons of categorical variables were performed 
using chi-square test $\left(\chi^{2}\right)$. The associations between the primary exposure factors and grades I-III DD were examined using adjusted logistic regression analysis and results reported as odds ratio (OR) with $95 \%$ confidence interval $(95 \% \mathrm{Cl})$. The associations between the primary exposure factors with individual parameters of $D D$ (E/e' ratio and LAVI) were evaluated using linear regression analysis. Covariates (age, gender, medication use, history of previous CVD, waist:hip circumference, BMI, pre-intervention eGFR and pre-intervention cholesterol profile) with $\mathrm{p}<0.05$ in the univariate models were included in the multivariableadjusted models. P-values of $<0.05$ in two-tailed testing were considered statistically significant. Statistical analyses were undertaken using SPSS version 18.0 for Windows (Statistical Package for Social Sciences; SPSS Inc, Chicago, IL).

\section{Results}

\section{Study Population}

The mean age was $(55.3 \pm 13.4)$ years and $73 \%$ were male. Prevalent CVD and hypertension were present in $6(7.6 \%)$ and 70 (90\%) subjects respectively. At least $50 \%$ were on either statin and/or ACE-inhibitor/ARB therapy. The median (IQR) GFR was 38 (27 43) $\mathrm{mL} / \mathrm{min} / 1.73 \mathrm{~m}^{2}$ and 25 subjects (32\%) had stage 4 CKD with median (IQR) GFR of 22 (19 27) $\mathrm{mL} / \mathrm{min} / 1.73 \mathrm{~m}^{2}$.

\section{Characteristics Of Participants With DD}

DD was present in 38 subjects (49\%) with grades I, II and III DD present in 14 (18\%), $21(27 \%)$ and $3(4 \%)$ of participants respectively. Compared to those without DD, subjects with DD were significantly older $(61.0 \pm 1.9$ vs $50.2 \pm 2.0 y r s ; p<0.001)$ and were more likely to be male ( $84 \%$ vs $63 \%$; $\mathrm{p}=0.04)$. Subjects with DD had higher 24 -hour PP $[48(95 \% \mathrm{Cl} 45,52)$ vs $43(95 \% \mathrm{Cl} 41,45) ; p=0.004]$ but had similar serum levels of IL-12, IL-18 and hsCRP (Table 1). 
There was a significant association between 24-hour PP and DD in the unadjusted model $(\mathrm{OR}=1.1 ; 95 \% \mathrm{Cl} 1.01,1.13)$. This was no longer significant $(\mathrm{OR}=1.03,95 \% \mathrm{Cl} 0.97,1.10)$ in the model adjusted for age (Table 2).

24-hour PP was significantly associated with severe DD in an unadjusted model $(\mathrm{OR}=1.08,95 \% \mathrm{Cl} 1.02,1.15)$. This was no longer significant $(\mathrm{OR}=1.06,95 \% \mathrm{Cl} 0.99,1.13)$ in a model adjusted for age (data not shown).

There was a significant association between 24-hour PP with the $E / e^{\prime}$ ratio $(B=0.01$; $p=0.005)$ in an unadjusted model. This relationship was no longer significant $(B=0.01$; $p=0.08$ ) in a model adjusted for age (data not shown). A significant association between 24hour PP and the LAVI $(B=0.01 ; p<0.001)$ in an unadjusted model remained significant $(B=0.7$; $\mathrm{p}<0.001$ ) after adjusting for age, previous CVD and BMI (data not shown).

\section{Association Between IL-12, IL-18 \& hsCRP With DD}

There were no significant associations between IL-12 (OR=0.99; $95 \% \mathrm{Cl} 0.98,1.01)$, IL$18(\mathrm{OR}=0.99 ; 95 \% \mathrm{Cl} 0.98,1.01)$ or hsCRP $(\mathrm{OR}=0.98 ; 95 \% \mathrm{Cl} 0.86,1.1)$ with DD in unadjusted or adjusted models (Table 2).

There were no significant associations between IL-12 (OR=1.0; 95\% $\mathrm{Cl} 0.98,1.01)$, IL$18(\mathrm{OR}=0.99 ; 95 \% \mathrm{Cl} 0.98,1.0)$ or hsCRP $(\mathrm{OR}=0.98 ; 95 \% \mathrm{Cl} 0.85,1.1)$ with severe DD (Grades II and III) in unadjusted or adjusted models (data not shown).

There was no association between IL-12 with the $E / e^{\prime}$ ratio $[B=(-0.04) ; p=0.1]$ or the LAVI $[B=(-0.01) ; p=0.7]$ in the unadjusted models. There was no association between IL-18 with the $E / e$ ratio $[B=(-0.06), p=0.1]$ or the $L A V I[B=(-0.02) ; p=0.6]$ in the unadjusted models. There was no association between hsCRP with the $E / e^{\prime}$ ratio $(B=0.03 ; p=0.4)$ or the LAVI $(B=0.02 ; p=0.5)$ in the unadjusted models. 


\section{Discussion}

Subclinical DD was present in almost $50 \%$ of patients with moderate CKD and was severe in over $60 \%$ of these. There was no association between pro-inflammatory cytokines and markers with DD. 24-hour PP was associated with LAVI and DD but this was no longer significant after adjustment for age.

Our study findings are similar to other CKD cohorts which demonstrate a high prevalence of asymptomatic subclinical DD in CKD patients as well as a direct relationship between the incidence and severity of DD with incremental CKD stage. This has significant clinical implications given that no effective interventions for DD currently exist and highlights the importance of early identification of DD as a potential opportunity for improved CVD risk stratification in CKD patients ${ }^{5}$. In a cohort of 300 CKD patients, the reported prevalence of DD was 55\% and $70 \%$ in stage 3 and $4-5$ respectively and increased to $60 \%$ and $80 \%$ after 1 -year follow-up ${ }^{27}$. Similar findings have also been reported in other CKD cohorts ${ }^{28}$. Subclinical DD is also an established prognostic marker of poor outcomes in the general population and CKD patients. In a community study of 2402 participants from the general population, severe DD was associated with a 10 -fold increased risk of all-cause mortality after adjustment for traditional risk factors ${ }^{8}$. Similarly, severe DD in a cohort of 129 chronic haemodialysis patients was associated with a 2.8- and 2.2-fold increased risk of mortality and CVD events respectively ${ }^{7}$.

The association between age, PP and DD has reportedly been inconsistent with cohort studies demonstrating both a lack of association between age and DD and that the association between PP and DD occurred independent of age ${ }^{15,29}$. This lack of association may reflect dissimilar patient characteristics across study cohorts which contribute to the uncertainty of the association between age and development of DD. Therefore, generalisibility of study findings attempting to evaluate this association across cohorts of varying risk and characteristics may not be appropriate. However, it is biologically plausible that the relationship between age and DD is mediated through the same mechanisms as that for PP. 
Nevertheless, this study adds to the body of knowledge regarding the relationship between arterial stiffness, PP and DD in CKD patients. There is a relative paucity of data in CKD with only two studies having demonstrated a potential relationship between arterial stiffness and DD in patients with CKD and end-stage kidney disease respectively ${ }^{15,30}$. This is clinically relevant as CKD is a disease population with disproportionately increased CVD risk causing excess mortality and significant socioeconomic burden due to associated morbidityrelated treatment costs. Given that current treatments have not reduced CVD-related mortality or morbidity in CKD patients there is urgent need to identify other potentially modifiable risk factors for DD such as arterial stiffness in prospective studies with large cohorts $^{2,31}$.

The utilisation of different methods to measure arterial stiffness and varying patient characteristics has also raised questions as to which measurement of arterial stiffness is optimally related to DD. In a community study of participants without CKD, aortic PWV had a stronger relationship with DD than peripherally measured $\mathrm{PP}^{29}$. Aortic $\mathrm{PWV}$ is considered the gold-standard non-invasive measurement of large artery stiffness while peripheral PP is potentially influenced by other factors such as heart rate, height and properties of the peripheral arteries ${ }^{13,29,32}$. A positive relationship between DD and clinic measured PP index has also recently been reported in a cohort of 511 patients with stage $3-4$ CKD $^{15}$. Significant differences between our study cohorts in terms of age, diabetes, blood pressure, use of ACE/ARBs, assessment of PP (24hour-PP versus clinic PPI) and diagnostic criteria for DD could also account for our conflicting results. There is evidence that specific antihypertensive classes such as ACE-inhibitors/angiotensin receptor blockers and calcium channel blockers preferentially lower central blood pressure, PP and arterial stiffness ${ }^{33}$. However, there was no association between class of anti-hypertensive agent and 24-hour PP in this study.

Chronic inflammation is associated with increased CVD in CKD patients with each increment in serum hsCRP being associated with a 1.21-fold increased risk of CVD mortality in chronic dialysis patients ${ }^{14,34}$. IL-12 and IL-18 are pro-atherogenic cytokines which are associated with increased CVD risk in the general population and are present at elevated concentrations in the sera of CKD patients ${ }^{16,17,19}$. However, evidence is conflicting as to whether IL-12 and IL-18 are similarly associated with CVD in CKD patients and their 
relationship with DD in this population has also not previously been explored ${ }^{19,21}$. Inflammation is implicated in the pathogenesis of DD as part of a reactive immunological response to persistent cardiomyocyte injury resulting in release of pro-fibrotic factors such as transforming growth factor(TGF) $-\beta^{12}$. In contrast to our study findings, the CRIC study reported a 1.14-fold increased risk of DD with serum hsCRP levels but not other proinflammatory cytokines traditionally associated with atherosclerosis such as IL-6 ${ }^{6}$. Serum hsCRP levels were slightly lower in our cohort (mean $<2 \mathrm{~g} / \mathrm{L}$ ) compared to the CRIC cohort (mean 2-3g/L) while IL-12 and IL-18 were similar to levels published in other CKD cohorts ${ }^{19}$. The relationship between inflammation and DD may also potentially have been modified by the cardio-protective and anti-inflammatory effects of statins or ACE/ARB which were prescribed to over $80 \%$ of our cohort ${ }^{35,36}$. Finally, failure to demonstrate a relationship between pro-atherogenic cytokines and DD in our studies could also reflect the separate and independent disease biology of DD from that of atherosclerosis ${ }^{5,37}$.

Limited sample size and short follow-up period may have led to erroneous inference and therefore we were unable to generate reliable estimates with certainty. Exclusion of participants with diabetes or current smoking may also have led to significant selection bias. Unmeasured confounders such as duration of CKD or hypertension may also have affected our findings.

\section{Conclusion}

Asymptomatic subclinical DD was present in $50 \%$ of a cohort of stage 3-4 CKD patients but was not associated with IL-12, IL-18 or hsCRP. The association between 24-hour PP and DD was no longer apparent following adjustment for age but given the small sample size our findings will need to be explored in larger sized cohorts of individuals with moderate stage CKD.

\section{Acknowledgements}

We thank Jackie Mansour, Christine Cowpland, Noeline Atkins and Lynette McCahon for diet counselling, nursing and technical assistance. We thank the renal physicians from 
Royal Perth Hospital (Dr. Mark Thomas, Dr. Barry Saker, Dr. Kevin Warr), Fremantle Hospital

(Dr. Paolo Ferrari, Dr. Helen Rhodes, Dr. Hemant Kulkarni) and Sir Charles Gairdner Hospital

(Dr. Brian Hutchison, Dr. Harry Moody, Dr. Grant Luxton, Dr. Steven Richards) for assistance in recruitment of patients.

\section{References}

1 Foley RN, Parfrey PS, Sarnak MJ. Clinical epidemiology of cardiovascular disease in chronic renal disease. American journal of kidney diseases : the official journal of the National Kidney Foundation. 1998; 32: S112-9.

2 Sarnak MJ, Levey AS, Schoolwerth AC, Coresh J, Culleton B, Hamm LL, et al. Kidney disease as a risk factor for development of cardiovascular disease: a statement from the American Heart Association Councils on Kidney in Cardiovascular Disease, High Blood Pressure Research, Clinical Cardiology, and Epidemiology and Prevention. Circulation. 2003; 108: 2154-69.

3 United States Renal Data System. USRDS Annual Report. Minneapolis: USRDS Registry 2012. Volume 1 CKD. Available from http://www.usrds.org/2012/pdf/v1 ch4 12.pdf.

4 Foley RN, Parfrey PS, Harnett JD, Kent GM, Martin CJ, Murray DC, et al. Clinical and echocardiographic disease in patients starting end-stage renal disease therapy. Kidney international. 1995; 47: 186-92.

5 Pecoits-Filho R, Bucharles S, Barberato SH. Diastolic heart failure in dialysis patients: mechanisms, diagnostic approach, and treatment. Seminars in dialysis. 2012; 25: 35-41.

6 Gupta J, Dominic EA, Fink JC, Ojo AO, Barrows IR, Reilly MP, et al. Association between Inflammation and Cardiac Geometry in Chronic Kidney Disease: Findings from the CRIC Study. PLoS One. 2015; 10: e0124772.

7 Barberato SH, Bucharles SG, Sousa AM, Costantini CO, Costantini CR, Pecoits-Filho R. [Prevalence and prognostic impact of diastolic dysfunction in patients with chronic kidney disease on hemodialysis]. Arquivos brasileiros de cardiologia. 2010; 94: 457-62.

8 Redfield MM, Jacobsen SJ, Burnett JC, Jr., Mahoney DW, Bailey KR, Rodeheffer RJ. Burden of systolic and diastolic ventricular dysfunction in the community: appreciating the scope of the heart failure epidemic. JAMA : the journal of the American Medical Association. 2003; 289: 194-202.

9 Mall G, Huther W, Schneider J, Lundin P, Ritz E. Diffuse intermyocardiocytic fibrosis in uraemic patients. Nephrology, dialysis, transplantation : official publication of the European Dialysis and Transplant Association - European Renal Association. 1990; 5: 39-44.

10 Abhayaratna WP, Barnes ME, O'Rourke MF, Gersh BJ, Seward JB, Miyasaka Y, et al. Relation of arterial stiffness to left ventricular diastolic function and cardiovascular risk prediction in patients $>$ or $=65$ years of age. The American journal of cardiology. 2006; 98: 1387-92.

11 Williams ES, Shah SJ, Ali S, Na BY, Schiller NB, Whooley MA. C-reactive protein, diastolic dysfunction, and risk of heart failure in patients with coronary disease: Heart and Soul Study. Eur J Heart Fail. 2008; 10: 63-9.

12 Glezeva N, Baugh JA. Role of inflammation in the pathogenesis of heart failure with preserved ejection fraction and its potential as a therapeutic target. Heart failure reviews. 2014; 19: 681-94.

13 Briet $M$, Boutouyrie $P$, Laurent $S$, London GM. Arterial stiffness and pulse pressure in CKD and ESRD. Kidney international. 2012; 82: 388-400.

14 Wanner C, Zimmermann J, Schwedler S, Metzger T. Inflammation and cardiovascular risk in dialysis patients. Kidney international. 61: S99-S102.

15 Lee WH, Hsu PC, Chu CY, Chen SC, Su HM, Lin TH, et al. Associations of pulse pressure index with left ventricular filling pressure and diastolic dysfunction in patients with chronic kidney disease. Am J Hypertens. 2014; 27: 454-9. 
16 Blankenberg S, Tiret L, Bickel C, Peetz D, Cambien F, Meyer J, et al. Interleukin-18 is a strong predictor of cardiovascular death in stable and unstable angina. Circulation. 2002; 106: 24-30.

17 Hansson GK, Libby P. The immune response in atherosclerosis: a double-edged sword. Nature reviews Immunology. 2006; 6: 508-19.

18 Fernandes JL, Mamoni RL, Orford JL, Garcia C, Selwyn AP, Coelho OR, et al. Increased Th1 activity in patients with coronary artery disease. Cytokine. 2004; 26: 131-7.

19 Yong K, Ooi EM, Dogra G, Mannion M, Boudville N, Chan D, et al. Elevated interleukin-12 and interleukin-18 in chronic kidney disease are not associated with arterial stiffness. Cytokine. 2013; 64: 39-42.

20 Liu YW, Su CT, Chang YT, Tsai WC, Su YR, Wang SP, et al. Elevated serum interleukin-18 level is associated with all-cause mortality in stable hemodialysis patients independently of cardiac dysfunction. PLoS One. 2014; 9: e89457.

21 Porazko T, Kuzniar J, Kusztal M, Kuzniar TJ, Weyde W, Kuriata-Kordek M, et al. IL-18 is involved in vascular injury in end-stage renal disease patients. Nephrology, dialysis, transplantation : official publication of the European Dialysis and Transplant Association - European Renal Association. 2009; 24: 589-96.

22 Mori TA, Burke V, Puddey I, Irish A, Cowpland CA, Beilin L, et al. The effects of [omega]3 fatty acids and coenzyme Q10 on blood pressure and heart rate in chronic kidney disease: a randomized controlled trial. Journal of hypertension. 2009; 27: 1863-72.

23 Levey AS, Bosch JP, Lewis JB, Greene T, Rogers N, Roth D. A more accurate method to estimate glomerular filtration rate from serum creatinine: a new prediction equation. Modification of Diet in Renal Disease Study Group. Annals of internal medicine. 1999; 130: 461-70.

24 Nagueh SF, Appleton CP, Gillebert TC, Marino PN, Oh JK, Smiseth OA, et al. Recommendations for the evaluation of left ventricular diastolic function by echocardiography. Journal of the American Society of Echocardiography : official publication of the American Society of Echocardiography. 2009; 22: 107-33.

25 Abhayaratna WP, Seward JB, Appleton CP, Douglas PS, Oh JK, Tajik AJ, et al. Left atrial size: physiologic determinants and clinical applications. Journal of the American College of Cardiology. 2006; 47: 2357-63.

26 Delanghe JR, Speeckaert MM. Creatinine determination according to Jaffe-what does it stand for? NDT plus. 2011; 4: 83-6.

27 Cai QZ, Lu XZ, Lu Y, Wang AY. Longitudinal changes of cardiac structure and function in CKD (CASCADE study). J Am Soc Nephrol. 2014; 25: 1599-608.

28 Park M, Hsu CY, Li Y, Mishra RK, Keane M, Rosas SE, et al. Associations between kidney function and subclinical cardiac abnormalities in CKD. J Am Soc Nephrol. 2012; 23: 1725-34.

29 Abhayaratna WP, Srikusalanukul W, Budge MM. Aortic stiffness for the detection of preclinical left ventricular diastolic dysfunction: pulse wave velocity versus pulse pressure. Journal of hypertension. 2008; 26: 758-64.

30 Fujiu A, Ogawa T, Matsuda N, Ando Y, Nitta K. Aortic arch calcification and arterial stiffness are independent factors for diastolic left ventricular dysfunction in chronic hemodialysis patients. Circulation journal : official journal of the Japanese Circulation Society. 2008; 72: 1768-72.

31 Gansevoort RT, Correa-Rotter R, Hemmelgarn BR, Jafar TH, Heerspink HJ, Mann JF, et al. Chronic kidney disease and cardiovascular risk: epidemiology, mechanisms, and prevention. Lancet. 2013; 382: 339-52.

32 Laurent S, Cockcroft J, Van Bortel L, Boutouyrie P, Giannattasio C, Hayoz D, et al. Expert consensus document on arterial stiffness: methodological issues and clinical applications. Eur Heart J. 2006; 27: 2588-605.

33 Dudenbostel T, Glasser SP. Effects of antihypertensive drugs on arterial stiffness. Cardiol Rev. 2012; 20: 259-63.

34 Stenvinkel P, Alvestrand A. Inflammation in end-stage renal disease: sources, consequences, and therapy. Seminars in dialysis. 2002; 15: 329-37. 
35 Ferrario CM, Strawn WB. Role of the renin-angiotensin-aldosterone system and proinflammatory mediators in cardiovascular disease. The American journal of cardiology. 2006; 98 : 121-8.

36 Ridker PM, Danielson E, Fonseca FA, Genest J, Gotto AM, Jr., Kastelein JJ, et al. Rosuvastatin to prevent vascular events in men and women with elevated C-reactive protein. The New England journal of medicine. 2008; 359: 2195-207.

37 Redfield MM. Heart Failure with Preserved Ejection Fraction. The New England journal of medicine. 2016; 375: 1868-77. 
Table 1. Characteristics Of Participants With Diastolic Dysfunction

\begin{tabular}{|c|c|c|c|}
\hline & Normal $(n=40)$ & $D D(n=38)$ & $p$-value \\
\hline Age (yrs) & $50.2 \pm 2.0$ & $61.0 \pm 1.9$ & $<0.001$ \\
\hline Gender (M, \%) & $26(63 \%)$ & $31(84 \%)$ & 0.04 \\
\hline CVD (n, \%) & $1(2 \%)$ & $5(14 \%)$ & 0.06 \\
\hline Statin $(n, \%)$ & $21(53 \%)$ & $18(49 \%)$ & 0.7 \\
\hline ACE/ARB (n, \%) & $38(95 \%)$ & $31(84 \%)$ & 0.1 \\
\hline BMI $\left(\mathrm{kg} / \mathrm{m}^{2}\right)$ & $27.1 \pm 1.0$ & $27.3 \pm 0.4$ & 0.8 \\
\hline Waist : Hip Ratio & $0.88 \pm 0.08$ & $0.93 \pm 0.01$ & 0.01 \\
\hline GFR $\left(\mathrm{mL} / \mathrm{min} / 1.73 \mathrm{~m}^{2}\right)$ & $37(29-43)$ & $39(23-43)$ & 0.6 \\
\hline Cholesterol (mmol/L) & $4.8(4.5,5.2)$ & $4.7(4.4,5.0)$ & 0.6 \\
\hline TG (mmol/L) & $1.6(1.3,1.9)$ & $1.7(1.5,2.0)$ & 0.6 \\
\hline LDL (mmol/L) & $2.5(2.2,2.8)$ & $2.6(2.3,2.9)$ & 0.7 \\
\hline HDL (mmol/L) & $1.4(1.3,1.5)$ & $1.2(1.1,1.3)$ & 0.02 \\
\hline \multicolumn{4}{|c|}{ 24-hour BP } \\
\hline Mean 24SBP (mmHg) & $118(116,120)$ & $121(117,126)$ & 0.1 \\
\hline Mean 24DBP (mmHg) & $74(72,76)$ & $73(69,76)$ & 0.3 \\
\hline Mean 24PP (mmHg) & $43(41,45)$ & $48(45,52)$ & $<0.005$ \\
\hline \multicolumn{4}{|c|}{ Inflammation } \\
\hline hs-CRP (mg/L) & $1.6(1.1,2.2)$ & $1.9(1.4,2.4)$ & 0.4 \\
\hline IL-12 (pg/mL) & $258(206-398)$ & $241(81-415)$ & 0.3 \\
\hline IL-18 (pg/mL) & $531(361-682)$ & $397(224-637)$ & 0.2 \\
\hline \multicolumn{4}{|c|}{ Echocardiography } \\
\hline LVEF (\%) & $64 \pm 1.2$ & $64 \pm 1.2$ & 0.8 \\
\hline LVMI $\left(g / m^{2}\right)$ & $82(78,87)$ & $92(86,98)$ & 0.01 \\
\hline LAVI $\left(\mathrm{mL} / \mathrm{m}^{2}\right)$ & $24(22,26)$ & $26(24,29)$ & 0.1 \\
\hline E/A ratio & $1.2(1.1,1.3)$ & $0.9(0.8,1.0)$ & $<0.001$ \\
\hline$e^{\prime}(\mathrm{cm} / \mathrm{s})$ & $10.3(9.7,10.9)$ & $7.6(7.0,8.1)$ & $<0.001$ \\
\hline E/e' ratio & $6.9(6.5,7.3)$ & $9.1(8.3,10.1)$ & $<0.001$ \\
\hline $\mathrm{Ar}-\mathrm{A}(\mathrm{ms})$ & $15(11.5,19.8)$ & $20(13.5,29.2)$ & 0.2 \\
\hline
\end{tabular}


24SBP (Mean 24-hour systolic blood pressure), 24DBP (Mean 24-hour diastolic blood pressure), 24PP (Mean 24-hour pulse pressure), hsCRP (highly sensitive C-reactive protein), IL-12 (Interleukin-12), IL-18 (Interleukin-18), LVEF (Left ventricular ejection fraction), LVMI (Left ventricular mass index), LAVI (Left atrial volume index) 
Table 2. Univariate \& Multivariate Analysis DD

\begin{tabular}{ccccccc}
\hline & & Univariate & & \multicolumn{2}{c}{ Multivariate } \\
\hline Age & OR & $95 \% \mathrm{Cl}$ & p-value & OR & $95 \% \mathrm{Cl}$ & $\mathrm{p}-\mathrm{value}$ \\
\hline 24PP & 1.1 & $(1.03,1.12)$ & 0.001 & 1.07 & $(1.02,1.12)$ & 0.01 \\
\hline CVD & 1.1 & $(1.01,1.13)$ & 0.02 & 1.03 & $(0.97,1.10)$ & 0.31 \\
\hline IL-12 & 6.3 & $(0.70,56.2)$ & 0.10 & - & - & - \\
\hline IL-18 & 0.99 & $(0.98,1.01)$ & 0.26 & - & - \\
\hline hs-CRP & 0.99 & $(0.98,1.01)$ & 0.21 & - & - \\
\hline
\end{tabular}

